THE ASTROPHYSICAL JOURNAL, $451: 477-483,1995$ October 1 (f): 1995. The American Astronomical Sociely. All rights reserved. Printed in USA.

\title{
INTERGALACTIC HELIUM ABSORPTION TOWARD HIGH-REDSHIFT QUASARS
}

\author{
Mark L. Giroux, Mark A. Fardal, ${ }^{1}$ and J. Michael Shull ${ }^{1}$ \\ Joint Institute for Laboratory Astrophysics, University of Colorado and National Institute of Standards and Technology, \\ Campus Box 440, Boulder, CO 80309 \\ Received 1994 November 18; accepted 1995 April 6
}

\begin{abstract}
The recent Hubble Space Telescope (HST) observations of the $z_{q}=3.286$ quasar Q0302-003 (Jakobsen et al. 1994) and the $z_{q}=3.185$ quasar Q1935 -67 by Tytler (1995) show absorption edges at the redshifted wavelength of $\mathrm{He}$ in $304 \AA$. A key goal is to distinguish between contributions from discrete Ly $\alpha$ forest clouds and a smoothly distributed intergalactic medium (IGM). We model the contributions from each of these sources of $\mathrm{He}$ II absorption, including the distribution of line Doppler widths and column densities, the "He II proximity effect " from the quasar, and a self-consistent derivation of the He II opacity of the universe as a function of the spectrum of ionizing sources, with the assumption that both the clouds and the IGM are photoionized. The He II edge can be fully accounted for by $\mathrm{He}$ II line blanketing for reasonable distributions of line widths and column densities in the $\operatorname{Lyx}$ forest, provided that the ionizing sources have spectral index $\alpha_{s}>1.5$, and any $\mathrm{He}$ II proximity effect is neglected. Even with some contribution from a diffuse IGM, it is difficult to account for the edge observed by Jakobsen et al. (1994) with a "hard" source spectrum $\left(\alpha_{s}<1.3\right)$. The proximity effect modifies the relative contributions of the clouds and IGM to $\tau_{\mathrm{He}}$ II near the quasar $\left(z \leqslant z_{q}\right)$ and markedly increases the amount of $\mathrm{He}$ II absorption required. This implies, for example, that to account for the He if edge with line blanketing alone, the minimum spectral index $\alpha_{s}$ must be increased from 1.5 to 1.9 . We demonstrate the need for higher resolution observations that characterize the change in transmission as $z \rightarrow z_{q}$ and resolve line-free gaps in the continuum. We set limits on the density of the diffuse IGM and suggest that the IGM and Ly $\alpha$ clouds are likely to be a significant repository for dark baryons.
\end{abstract}

Subject headings: galaxies: evolution - galaxies: intergalactic medium - quasars: absorption lines

\section{INTRODUCTION}

The Hubble Space Telescope Faint Object Camera (FOC) detected (Jakobsen et al. 1994) redshifted He II $\lambda 304$ absorption, with optical depth $\tau_{\mathrm{He} I \mathrm{I}}=3.2_{-1.1}^{+\infty}$, in the spectrum of the $z=3.286$ quasar $\mathrm{Q} 0302-003$. This absorption may represent the Gunn-Peterson (GP) effect (Gunn \& Peterson 1965) in a diffuse intergalactic medium (IGM), although, owing to the low resolution of the spectrum, it was not possible to distinguish He II absorption from a uniformly distributed IGM from line blanketing in the Ly $\alpha$ clouds. More recently, Tytler et al. (1995) reported that the Hubble Space Telescope Faint Object Spectrograph (FOS) detected absorption with optical depth $\tau_{\mathrm{He} \mathrm{II}}=1.0 \pm 0.2$ in the spectrum of the $z=3.185$ quasar Q1935-67. Such measurements provide powerful new constraints at $z \gtrsim 3$ on the thermodynamic state of the IGM, on the spectra of ionizing sources, and on the nature of the " $\mathrm{Lyx}$ forest "(LF) of discrete $\mathrm{H}$ I absorption lines.

The presence of $\mathrm{He}$ II absorption along the sight lines of quasars whose spectra have radiation down to $(304 \AA)(1+z)$ is a result which has been anticipated (see Miralda-Escudé \& Ostriker 1992; Miralda-Escudé 1993; Miralda-Escudé \& Rees 1994; Shapiro, Giroux, \& Babul 1994). For models in which the IGM and/or LF clouds are photoionized, unless the spectrum of the sources is exceedingly hard, the $\mathrm{He} \mathrm{II} / \mathrm{H} 1$ ratio in the diffuse IGM and the clouds may be quite large, and the attendant $\mathrm{He}$ II absorption will exceed the observed low level of H I absorption. As noted by Miralda-Escudé (1993), simple assumptions about the nature of the ionizing sources (quasars) and the $L F$ distribution could imply $\tau_{\mathrm{He}} \sim 1$ from clouds

\footnotetext{
${ }^{1}$ Also at Center for Astrophysics and Space Astronomy, Department of Astrophysical, Planetary, and Atmospheric Sciences University of Colorado.
}

alone. Thus the relative contributions of the IGM and the LF to the observed $\mathrm{He}$ II absorption are an important further constraint.

Jakobsen et al. (1994) used the extensive statistics on the LF distribution to estimate the line-blanketing contribution to the absorption. Assuming that all lines were thermally broadened, they found that even if they were to extend the distribution to $N_{\mathrm{HI}} \approx 10^{12} \mathrm{~cm}^{-2}$, a ratio $\mathrm{He} \mathrm{II} / \mathrm{H}_{\mathrm{I}} \approx 2000$ was required for line blanketing to account for the absorption. This would require an ionizing background with a substantial component of soft ionizing sources. Since the distribution (or existence) of clouds with $N_{\mathrm{HI}}<10^{12} \mathrm{~cm}^{-2}$ is not established (at those column densities the distinction between rarefied clouds and IGM becomes blurred) they concluded that for a harder active galactic nucleus (AGN) ionizing spectrum, substantial absorption from a diffuse IGM must be present. Madau \& Meiksin (1994) pointed out that the observed He II trough may be produced entirely by line blanketing if the clouds are velocity broadened and/or their distribution extends to very low column densities, $N_{\mathrm{HI}} \approx 2 \times 10^{12} \mathrm{~cm}^{-2}$, in a soft ionizing background. The opacity of intervening clouds may naturally soften the background spectra of AGNs (Miralda-Escudé \& Ostriker 1990). Songaila, Hu, \& Cowie (1995) argue that including the lowest column density clouds may be unnecessary. From a high resolution, high signal-to-noise spectrum obtained using the HIRES spectrograph on the Keck $10 \mathrm{~m}$ telescope, they measured the $\mathrm{H}_{1}$ Ly $\alpha$ forest in the spectrum of $\mathrm{Q} 0302-003$. They conclude that a ratio $\mathrm{He} \mathrm{II} / \mathrm{H} \mathrm{I} \approx 80$ implies $\tau_{\mathrm{He} \text { II }}=1.6$, without even including the absorption associated with the weakest $\mathrm{H}$ I lines.

In this paper, we make a theoretical computation of the He II line blanketing toward the two lines of sight. We explore 
several important effects: (1) the distribution of Doppler widths ( $b$-values) in the Ly $\alpha$ clouds; (2) the distribution of $\mathrm{H}$ I column densities; (3) the self-consistent "filtering" of the metagalactic ionizing radiation field by the $L y \alpha$ clouds; and (4) the local photoionizing effects of the quasar (the " $\mathrm{He}$ II proximity effect") on both the LF clouds and the diffuse IGM. These processes are all significant, especially that of quasar proximity, which can be used to distinguish line blanketing from diffuse IGM absorption. Zheng \& Davidsen (1995) also considered the consequences of the $\mathrm{He}$ II proximity effect on the absorption seen toward high-redshift QSOs. However, they have restricted their analysis to the enhanced ionization of $\mathrm{He}$ II in the diffuse IGM. As we show, the local radiation field of the quasar will have a markedly different effect on absorption due to the Lya forest.

Even at $z \geq 3$, the He II line blanketing should be patchy, and line-free gaps should appear, characteristic of the relative optical depths in the IGM and Ly $\alpha$ clouds. These new He II observations can be used to constrain the cloud distributions in $N_{\mathrm{H} 1}$, the metagalactic ionizing spectrum, and the contribution, $\Omega_{1 G M}$, of the IGM to the baryonic closure parameter. As a result, we consider the diffuse IGM as a possible repository for a significant number of dark baryons.

\section{THE CONTINUUM AND LINE OPACITY OF THE LY $\alpha$ FOREST}

The choice of a model for the distribution, $\partial^{2} N_{c} / \partial N_{\mathrm{HI}} \partial_{z}$, of the Ly $\alpha$ clouds in column density and redshift has the twofold effect of determining the level of the line blanketing and the degree of filtering of the radiation background. The continuum optical depth of the LF for a random spatial distribution is given by (Paresce, MeKee, $\&$ Bowyer 1980)

$$
\frac{d \tau(v)}{d z}=\int_{0}^{\infty} \frac{\partial^{2} N_{\mathrm{c}}}{\partial N_{\mathrm{H} 1} \partial z}\left[1-e^{N_{\mathrm{HI} \sigma_{\mathrm{eff}}(v)}}\right] d N_{\mathrm{H} 1}
$$

where, for clouds composed of $\mathrm{H}$ and $\mathrm{He}$,

$$
\sigma_{\text {eff }}(v)=\sigma_{\mathrm{H} \mathrm{I}}(v)+\left[\frac{N_{\mathrm{HeI}}}{N_{\mathrm{HI}}}\right] \sigma_{\mathrm{He} \text { I }}(v)+\left[\frac{N_{\mathrm{He} \mathrm{II}}}{N_{\mathrm{H} 1}}\right] \sigma_{\mathrm{He} \mathrm{II}}(v) .
$$

The wavelength-averaged contribution to the line optical depth due to (LF) He II resonant scattering of radiation may be written (Møller \& Jakobsen 1990)

$$
\tau_{\mathrm{LF}}(z)=\frac{d N(z)}{d z} \frac{\left\langle W_{\dot{\lambda}}\right\rangle}{\lambda}(1+z),
$$

where $d N / d z$ is the number of lines per unit redshift and $\left\langle W_{\lambda}\right\rangle$ is the mean equivalent width of $\mathrm{He}$ II $\lambda 304$ lines in LF systems averaged over their distribution in column density.

The distribution of the LF clouds has often been parameterized by a function of the form $\partial^{2} N_{c} / \partial N_{\mathrm{HI}} \partial_{z}=$ $A(1+z)^{\gamma} N_{\mathrm{H}}^{-\beta}$ for $N_{\mathrm{r}}(\mathrm{H} \mathrm{I}) \leq N_{\mathrm{H} 1}\left(\mathrm{~cm}^{-2}\right) \leq N_{\mathrm{u}}(\mathrm{H}$ I). Our chosen parameters $\left(A=2.7 \times 10^{6}, \gamma=2.46, \beta=1.43\right)$ are taken from the study by Press \& Rybicki (1993, hereafter denoted PR) of the depression due to the Lyman series lines in the averaged spectrum of many high-redshift quasars. Their model has the useful property that it includes an estimate of the distribution in Doppler width $b$, which we use in solving for the contribution of the clouds to line blanketing. It produces a more generous estimate of the line blanketing than that adopted by Jakobsen et al. (1994). For example, the PR distribution produces an $H$ I absorption $\tau_{\mathrm{HI}} \approx 0.44$, compared to $\tau_{\mathrm{HI}}=0.35$ \pm 0.1 inferred from observations of $\mathrm{H} 1$ absorption at $z=3.3$
(Steidel \& Sargent 1987; Schneider, Schmidt, \& Gunn 1991; Webb et al. 1992). In general, our estimates of the contribution of the diffuse IGM to the He II absorption will be conservative.

Since, for $1 \leq \beta \leq 2$, clouds with $N_{\mathrm{H}} \approx \sigma_{\text {eff }}^{-1}$ dominate the continuum opacity, the continuum optical depth $\tau(z)$ is sensitive to the upper limit $N_{u}(\mathrm{H}$ I) in the LF. On the other hand, the He II LF optical depth $\tau_{\mathbf{L F}}$ is dominated by mildly saturated lines with $N_{\mathrm{He} \mathrm{II}} \approx 10^{14} \mathrm{~cm}^{-2}$. Since we will typically find that the ratio $\eta \equiv N_{\mathrm{He} \mathrm{II}} / N_{\mathrm{HI}} \gtrsim 60$, the He II optical depth is more sensitive to the lower limit $N_{N}(\mathrm{H}$ I). PR state that their fit is good from at least $N_{t}(\mathrm{H} \mathrm{I})=10^{13} \mathrm{~cm}^{-2}$ to $N_{u}(\mathrm{H} \mathrm{I})=10^{16}$ $\mathrm{cm}^{-2}$, but in view of the likely existence of clouds at even lower $H$ I columns, we also explore the effects of varying these limits. Lyman-limit absorption systems with $N_{\mathrm{H} 1} \geq 10^{17} \mathrm{~cm}^{-2}$ are an additional source of continuum opacity. For these clouds, we use a distribution based on the observations of Sargent, Steidel, \& Boksenberg (1989, hereafter denoted SSB), extrapolated down to $N_{\mathrm{HI}}=10^{17} \mathrm{~cm}^{-2}$ with $A=1.8 \times 10^{6}, \beta=1.39$, and $\gamma=0.68$.

An estimate of the He II continuum and line opacity depends critically on the $\mathrm{He} \mathrm{II} / \mathrm{H}$ I ratio $\eta$ in the clouds. In highly photoionized clouds,

$$
\eta \approx\left(\frac{\alpha_{\mathrm{He} \mathrm{II}}}{\alpha_{\mathrm{H} 1}}\right)\left(\frac{\Gamma_{\mathrm{HI}}}{\Gamma_{\mathrm{He} \mathrm{II}}}\right)\left(\frac{n_{\mathrm{He}}}{n_{\mathrm{H}}}\right) \approx 1.7\left(\frac{J_{\mathrm{HI}}}{J_{\mathrm{He} \mathrm{II}}}\right),
$$

where $\alpha_{\mathrm{He} \text { II }}$ and $\alpha_{\mathrm{H} \text { I }}$ are the radiative recombination rates to He II and $\mathrm{H}_{\mathrm{I}}$ at $T \approx 2 \times 10^{4} \mathrm{~K}$, and $\Gamma_{\mathrm{H} \text { I }}$ and $\Gamma_{\mathrm{He} \text { II }}$ are the photoionization rates for $\mathrm{H}_{\mathrm{I}}$ and $\mathrm{He} \mathrm{II}$, respectively. The quantities $J_{\mathrm{H} I}$ and $J_{\mathrm{He} \mathrm{I}}$ are the mean intensities at the ionizing thresholds $v_{\mathrm{H}}(1 \mathrm{ryd})$ and $v_{\mathrm{He} I \mathrm{I}}(4 \mathrm{ryd})$ and we assume $\mathrm{He} /$ $\mathbf{H}=0.08$ by number. This expression is coupled to the continuum opacity (eqs. [1] and [2]). If the opacities at $v_{\mathbf{H}}$ and $v_{\text {He II }}$ are large at $z>3$, the radiation transfer becomes localized, and a reasonable approximation (Giroux 1992; Giroux \& Shapiro 1995 ) is to express $J_{\mathrm{H} 1}$ and $J_{\mathrm{He} I l}$ as the source function, $S_{\mathrm{v}}=$ $j_{v} / \kappa_{v}$, where $j_{\mathrm{H} \text { I(He II) }}$ is the emissivity of the sources at $v_{\mathrm{HI}}$ (He II) and $\kappa_{\mathrm{H} 1\left(\mathrm{He} \mathrm{Ii}_{\mathrm{e}}\right)}$ is the opacity at $v_{\mathrm{H} \text { (He II) }}$ [here, $\left.\kappa=(d z / d t)(d \tau / d z)\right]$ $c]$. We may then write $\eta \approx 1.7\left(j_{\mathrm{H}} / j_{\mathrm{He} \mathrm{II}}\right)\left(\kappa_{\mathrm{He} \mathrm{II}}(\eta) / \kappa_{\mathrm{HI}}\right)$. Given the distribution of absorption line clouds, $\eta$ may be cvaluated self-consistently for any assumed ratio $\left(j_{\mathrm{H}} / j_{\mathrm{He} \mathrm{II}}\right)$ of ionizing sources. If $N_{l}=10^{13} \mathrm{~cm}^{-2}, N_{\mu}=10^{16} \mathrm{~cm}^{-2}$, and the source spectra are constant power laws with spectral index $\alpha_{s}$, we find $\eta \approx 6.5,97$, and 650 for $\alpha_{s}=1.0,1.5$, and 2.0 . After filtering by intervening absorption line clouds, the mean background spectral indices are 1.0,3.0, and 4.3. The steepening of background spectral indexes due to filtering by clouds is quite sensitive to $\eta$ and increases rapidly as the source spectra steepen from $\alpha_{s}=$ 1.0 to 1.5. While the assumption that the sources and the filtered background retain their power-law shape may not be highly accurate (Madau 1995), the nonlinear dependence of $J_{\mathrm{He} \mathrm{II}} / J_{\mathrm{H} / \mathrm{l}}$ on $j_{\mathrm{He} \mathrm{I}} / j_{\mathrm{H} /}$ is not strongly affected by the actual spectral shapes.

Figure 1 illustrates the sensitivity of $\mathrm{He}$ In line blanketing to line width and source spectra. These models assume $N_{1}=10^{13}$ $\mathrm{cm}^{-2}$ and $N_{u}=10^{16} \mathrm{~cm}^{-2}$ with PR's " $\Gamma$ distribution " in column density. We consider a set of single values for the Doppler width $b$, as well as the PR distribution in $b(\mathrm{H} \mathrm{I})$. We use two limiting assumptions about thermal and bulk motions within the clouds: (1) $b(\mathrm{He} \mathrm{I})=b(\mathrm{H} \mathrm{I})$ ("velocity broadening "); and (2) $b(\mathrm{He} \mathrm{II})=0.5 b(\mathrm{H} \mathrm{I})$ ("thermal broadening"). As Figure $1 a$ shows, the assumed $b$ for the $\mathrm{He}$ II lines may alter the line blanketing optical depth by as much as a factor of 2 when 


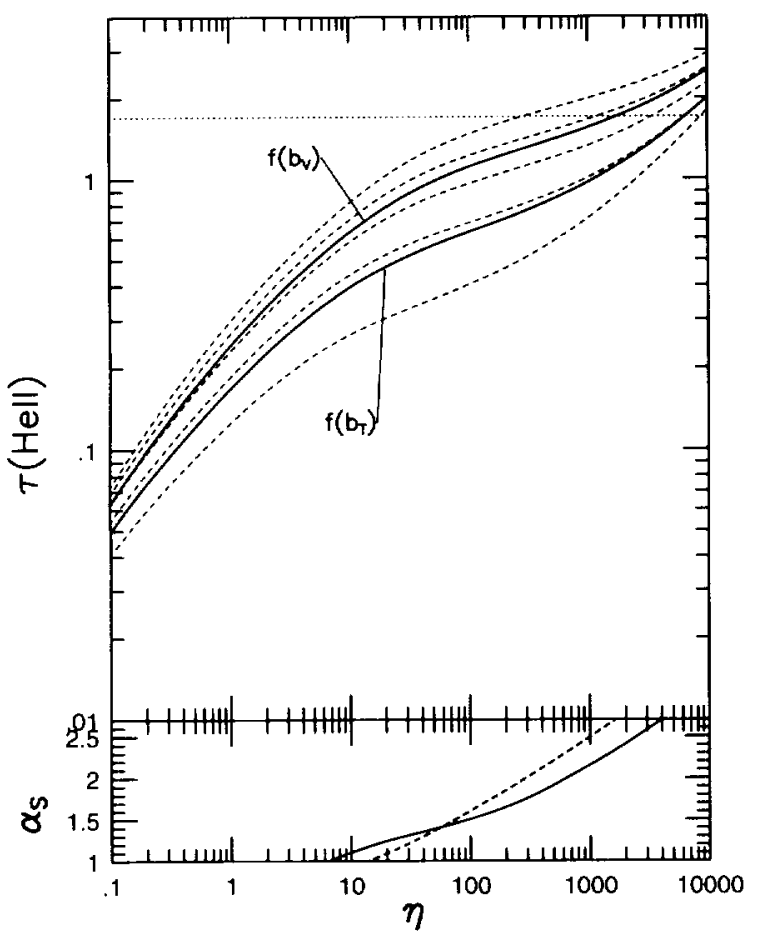

FIG. 1- (a) (top) He Il optical depth $\tau_{\mathrm{LF}}\left(\mathrm{He}\right.$ II) from $\mathrm{LF}$ vs. $\eta=N_{\mathrm{Hc}} / N_{\mathrm{H}}$ for Doppler widths (dashed curves, showing $b_{\mathrm{HII}}=10,20,30,40,50 \mathrm{~km} \mathrm{~s}^{-}$ from bottom to top). The horizontal dotted line shows the $\tau_{\mathrm{Hell}}=1.7$ limit toward Q0302-003. The two solid curves assume the PR distribution in $h\left(\mathrm{H}_{1}\right)$ with either velocity-broadened lines $\left(b_{\mathrm{He}}=b_{\mathrm{H}}\right)$ or thermally broadened lines $\left(b_{\mathrm{Hell}}=0.5 b_{\mathrm{H}}\right)$. All curves assume lower limit $N_{\mathrm{r}}(\mathrm{H} \mathrm{I})=10^{13} \mathrm{~cm}^{-2}$ and upper limit $N_{\mathrm{u}}(\mathrm{H} \mathrm{I})=10^{16} \mathrm{~cm}^{-2}$. (b) (bottom) Source spectral index $\alpha_{3}$ corresponding to $\eta$, assuming an upper limit on $N_{4}(\mathrm{H} 1)$ of either $10^{16} \mathrm{~cm}^{-2}$ (solid curve) or $10^{17} \mathrm{~cm}^{-2}$ (dashed curve), and $N_{(}(\mathrm{H})=10^{13} \mathrm{~cm}^{-2}$.

$\tau_{\mathrm{He} \mathrm{II}} \approx 1$. The required $\mathrm{He} \mathrm{II} / \mathrm{H}$ I ratio is extremely sensitive to the optical depth and depends on whether the clouds are assumed to be broadened by thermal or bulk motions (Madau $\&$ Meiksin 1994). From calculations that include the actual distribution in $b$, we find that an adequate estimate of the line blanketing may be obtained by using a single value $b(\mathrm{He}$ II) $\approx 17.5(35) \mathrm{km} \mathrm{s}^{-1}$ for thermal (velocity) broadening, consistent with the median values, $b(\mathrm{H} \mathrm{I})=26-33 \mathrm{~km} \mathrm{~s}^{-1}$, found by Rauch et al. (1992, 1993).

Figure $1 b$ shows the source spectral index needed to provide the required value of $\eta$. In general, $\eta>1000$ is required if only the clouds above $N_{l}(\mathrm{H} \mathrm{I})=10^{13} \mathrm{~cm}^{-2}$ are to account for the majority of the absorption. This value is still consistent with a source spectrum having $\alpha_{s} \lesssim 2.5$. However, Figure 2 shows (see Jakobsen et al. 1994) that the inclusion of lower column clouds has a strong effect on the $\mathrm{He}$ II line blanketing. The cases shown illustrate the effects of changing $N_{1}$ and $N_{u}$, assuming a PR distribution in $b$ and $N_{\mathrm{H}}$, and they demonstrate the dependence on the assumption of velocity or thermal broadening. Figures $2 a$ and $2 b$ show that if $\tau_{\mathrm{He} \mathrm{II}} \approx 1$, the line-blanketing opacity is relatively insensitive to the inclusion of higher column clouds, since their lines are saturated. If lower column clouds are included, the line blanketing opacity is enhanced; since $\eta$ is sensitive to the opacity for $\tau_{\mathrm{He} \|} \approx 1$, the required $\eta$ is significantly diminished. If the clouds are velocity broadened, and if clouds down to $N_{1}=10^{12} \mathrm{~cm}^{-2}$ are included, $\eta \sim 100$ is required if lines are to account for the majority of the absorption. This result is consistent with that of Madau \& Meiksin

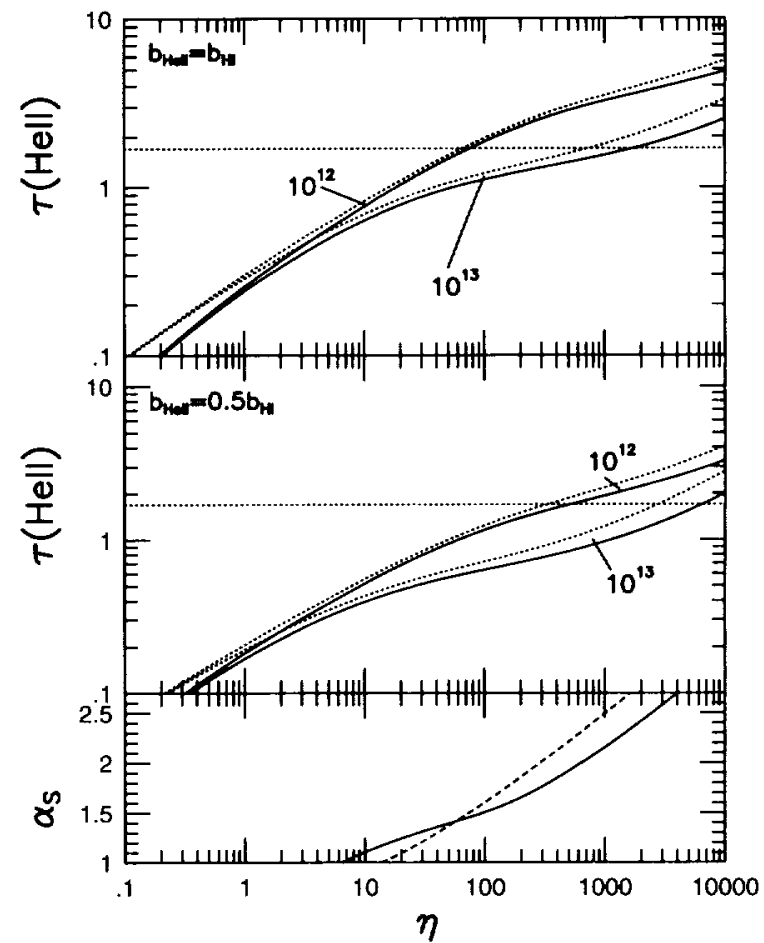

Fig. 2.- $-(a)$ (top) Effects of column-density distribution on $\tau_{\mathrm{Lf}}(\mathrm{He}$ il) assuming the PR distribution $f(b)$ and velocity broadening. Solid curves assume $N_{u}(\mathrm{H} \mathrm{I})=10^{16} \mathrm{~cm}^{-2}$; dashed curves assume $N_{u}\left(\mathrm{H} \mathrm{I}_{\mathrm{l}}\right)=10^{17} \mathrm{~cm}^{-2}$. The assumed lower limit $N(\mathrm{H})$ is either $10^{12}$ or $10^{13} \mathrm{~cm}^{-2}$ as labeied. (b) (middle) Same as (a), except that all lines are assumed to be thermally broadened. (c) (bottom) Source spectral index corresponding to $\eta$ as in Fig. $l b$.

(1994) but disagrees with the $\eta \geq 2000$ inferred by Jakobsen et al. (1994) for $N_{l}=10^{12} \mathrm{~cm}^{-2}$, primarily because they assumed thermal broadening and adopted a different opacity model. As Figure $2 c$ shows, for $\tau_{\mathrm{He} \text { II }} \approx 1$ from line blanketing alone, our results are consistent with sources with spectral indices $1.4 \lesssim$ $\alpha_{\mathrm{s}} \lesssim 2$

\section{RESULTS}

\subsection{The He II Proximity Effect}

Close to the quasar, the number density of atoms in a given ionization state, $n(X)$, is altered from that expected if it is photoionized solely by the metagalactic background radiation (MBR). Therefore,

$$
\frac{n(X)}{n_{\mathrm{mbr}}(X)} \approx \frac{4 \pi J_{\mathrm{mbr}}}{\left(4 \pi J_{\mathrm{mbr}}+f_{q}\right)} \equiv \frac{J_{\mathrm{mbr}}}{J_{\mathrm{tot}}},
$$

where the flux (ergs $\mathrm{cm}^{-2} \mathrm{~s}^{-1} \mathrm{~Hz}^{-1}$ ) from the quasar seen at a redshift $z_{\text {abs }}$ is

$$
f_{q}\left(z_{q}, z_{\mathrm{abs}}\right)=f_{0} \frac{\left(1+z_{\mathrm{abs}}\right)^{4}}{\left(1+z_{q}\right)}\left[\frac{1-\left(1+z_{q}\right)^{-1 / 2}}{\left(1+z_{\mathrm{abs}}\right)^{-1 / 2}-\left(1+z_{q}\right)^{-1 / 2}}\right]^{2},
$$

for $\Omega_{0}=1$ (Bajtlik, Duncan, \& Ostriker 1988). The flux measured at Earth from Q0302-003 is $f_{0} \approx 2 \times 10^{-27} \mathrm{ergs} \mathrm{cm}^{-2}$ $\mathrm{s}^{-1} \mathrm{~Hz}^{-1}$ at rest frequency $v_{\mathrm{H}}$ (see Fig. 1 in SSB) extrapolated to $v_{\mathrm{He} I I}$ assuming the same spectral index $\alpha_{s}$ as the average source spectrum. If the ionizing background arises from a combination of "thermal-bump" emission from AGN and/or hot stars, the spectrum of Q0302-003 may be locally harder, thus increasing the proximity effect. If the flux $f_{0}$ was measured 
during an AGN "flare," we are overestimating the quasar's luminosity. However, if such an effect is typical of bright AGN, it would correspondingly decrease the estimate of $J_{0}=\left(10^{-21}\right.$ ergs $\left.\mathrm{cm}^{-2} \mathrm{~s}^{-1} \mathrm{~Hz}^{-1} \mathrm{sr}^{-1}\right) J_{-21}$, the mean intensity of the radiation background at $v=v_{\mathbf{H}}$, and so perhaps leave the proximity effect unchanged. We adopt $J_{-21}=1$ throughout (Bechtold 1994).

The wavelength region affected by Q0302-003 depends both on an accurate measurement of the systemic redshift, $z_{q}$, of the quasar and on its luminosity. Tytler \& Fan (1992) found $z_{\mathrm{em}}=3.2899 \pm 0.0008$ from a maximum-likelihood analysis of Lyx, C IV, and Si IV + O IV], although the individual redshifts of Ly $\alpha(z=3.2859)$ and $C$ IV $(z=3.2864)$ argue for $z_{\mathrm{em}}=3.286$ (SSB). It is possible that $z_{\mathrm{q}}>z_{\mathrm{em}}$ owing to velocity shifts between lines from different ionization stages (Gaskell 1982; Espey et al. 1989; Corbin 1990) which would reduce the strength of the proximity effect at fixed wavelength. However P. Francis (1995) has pointed out to us that Q0302-003 has the brightest, narrowest Ly $\alpha$ line in the entire Bright Quasar Survey, and this line is probably at the systemic redshift. In our calculations we adopt $z_{q}=3.286$.

For a concrete example, consider the case of $\alpha_{s}=1.5, N_{l}=$ $10^{13} \mathrm{~cm}^{-2}$, and $N_{4}=10^{16} \mathrm{~cm}^{-2}$. In this case $J_{-21}(\mathrm{He} \mathrm{II)}=$ $1.7 J_{-21}(\mathrm{H} \mathrm{I}) / \eta \approx(1.7 / 97) J_{-21} \approx 0.017$, and the quasar's flux dominates the metagalactic background down to $1280 \AA$, just longward of the 1262-1275 $\AA$ void in the He in forest inferred from the observed void in the $H_{I}$ forest of the quasar (Dobrzycki \& Bechtold 1991). This inference depends on the relative contribution of low $\mathrm{H}$ I column lines below the sensitivity limits of Dobrzycki \& Bechtold (1991). The He II proximity effect extends farther in $\Delta z$ than that in $H_{I}$, since the metagalatic He II ionizing background is more filtered by the LF. Because the continuum opacity of the intervening clouds begin to soften the "bare" quasar spectrum when $\Delta z>0.15$ (observed-frame $\Delta \hat{\lambda} \approx 50 \AA$ ), we do not include this effect. Over this range, the averaged $\mathrm{He}$ II continuum optical depth due to the quasar-ionized clouds remains below unity, and the high column density clouds that dominate the average He II continuum opacity are unlikely to be present.

A key goal is to distinguish He II line absorption from that in a diffuse IGM (the GP effect). Figures 3 and 4 demonstrate the different behaviors of $\tau_{L F}$ and $\tau_{G P}$ near $z_{q}$. We denote the density of the diffuse IGM relative to the closure density by $\Omega_{\mathrm{IGM}}$. In order to scale this by factors which enter the same way for the $\mathrm{H}$ I and He II GP effects, we define

$$
\boldsymbol{\Omega}_{\mathrm{IGM}} \equiv h^{3 / 2} T_{4}^{-3 / 8}[(3+p) / 3]^{1 / 2} J_{-2 / 2}^{-1 / 2} \Omega_{\mathrm{IGM}} \text {. }
$$

Then the GP optical depth in He II from the IGM is

$$
\begin{aligned}
\tau_{\mathrm{GP}}(\mathrm{He} \text { II) } & =\left(1.15 \times 10^{9}\right) h^{-1} n_{\mathrm{He} \mathrm{II}}(z)\left(\frac{1+z}{4.29}\right)^{-3 / 2} \\
& =64.3 \eta \Omega_{\mathrm{lGM}}^{2}\left(\frac{J_{\mathrm{tot}}}{10^{-21}}\right)^{-1} .
\end{aligned}
$$

Here, $T_{4}$ is the IGM temperature in units of $10^{4} \mathrm{~K}, H_{0}=(100$ $\left.\mathrm{km} \mathrm{s}^{-1} \mathrm{Mpc}^{-1}\right) h$, and $p \approx 0$ (Madau \& Meiksin 1994) is the spectral slope in frequency immediately above the $\mathrm{H} I$ and $\mathrm{He}$ II ionization thresholds. Thus, the optical depth of the diffuse IGM, $\tau_{\text {GP }} \propto J_{\text {tot }}^{-1}$. In contrast, the LF optical depth $\tau_{L F} \propto$ $J_{\text {tot }}^{\beta-2} \approx J_{\text {tot }}^{-0.6}$ when the column densities of the saturated clouds lie within the allowed range $\left(N_{1}\right.$ to $\left.N_{u}\right)$, and $\tau_{L, F}$ is almost independent of $J_{\text {tot }}$ when they lie far below.

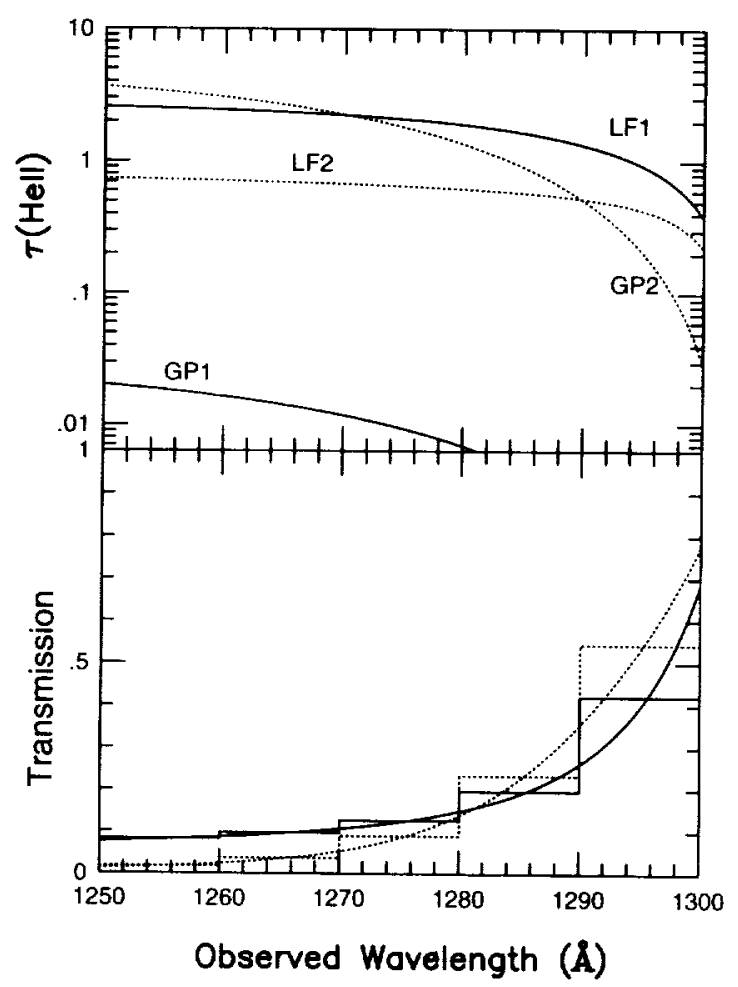

FIG. 3.- $-(a)(t o p) \mathrm{He}$ II optical depth from Ly $\alpha$ forest $\left[\tau_{\mathrm{LF}}(\mathrm{He}\right.$ II)] and diffuse $\operatorname{IGM}\left[\tau_{\mathrm{GP}}(\mathrm{He} I)\right]$ vs. wavelength in the spectrum of $\mathrm{Q0302}-003$, as the abundance of $\mathrm{He} 1 \mathrm{is}$ altered by ionizing radiation from the quasar. The redshifted He $I$ il $\lambda 303.78$ lies at $1302.0 \AA$ and the total opacity is assumed to be $\tau_{\text {Hc II }}=1.7$. Model 1 is chosen so that line blanketing is the dominant component of the opacity, while model 2 is chosen so that the diffuse IGM is the dominant component of the opacity. For model 1 (solid curves) the source spectral index $\alpha_{s}=1.94$, the background radiation produces $\eta=560$, the LF extends from $N_{1}=10^{12} \mathrm{~cm}^{-2}$ to $N_{4}=10^{16} \mathrm{~cm}^{-2}$, lines are velocity broadened with the PR distribution $f(b)$, and the scaled diffuse IGM density $\Omega_{\mathrm{IGM}}=0.001$. For model 2 (dashed curves) $x_{s}=1.85$, the background radiation produces $\eta=403$, the LF extends from $N_{1}=10^{13} \mathrm{~cm}^{-2}$ to $N_{u}=10^{16} \mathrm{~cm}^{-2}$, lines are thermally broadened with the PR distribution $f(b)$, and the scaled difluse IGM density $\Omega_{\mathrm{IGM}}=0.0152$. (b) (bottom) Corresponding transmission $T(\lambda)$ below the $304 \AA$ edge for models 1 (solid curve) and 2 (dotted curve).

Figure $3 a$ illustrates the changing GP and line optical depths toward Q0302-003 as $\lambda \rightarrow(303.784 \AA)\left(1+z_{q}\right) \approx 1302.0 \AA$ for two cases, one in which the optical depth is dominated by the line-blanketing contribution, and one in which the IGM contribution dominates. Figure $3 b$ shows the corresponding transmission in the wavelength region $1250-1300 \AA$ both continuously and in $10 \AA$ bins. The asymptotic (i.e., far from the quasar) optical depth for the models is chosen so that the mean transmission over this $50 \AA$ wavelength region is $\langle T\rangle=0.18$, consistent with the $90 \%$ confidence limit of $\tau_{\text {He II }}=1.7$ (Jakobsen et al. 1994). Given the large amount of noise in the existing spectrum and the wavelength uncertainty of the $H S T / F O C$, it is possible that a more rapid increase in transmission, such as that shown here, could be missed. Better signal-to-noise observations could show this effect. For the LF-dominated model, the asymptotic optical depth is 2.9, while for the IGM-dominated model the asymptotic optical depth is 6.8. If other lines of sight at $z>3$ have $\tau_{\mathrm{He} \mathrm{II}} \approx 1$, such as the object observed by Tytler et al. (1995), it becomes much easier to explain the absorption by line blanketing alone. Figure 4 shows the optical depths and transmission for cases analogous to that of Figure 3, but corresponding to the mean 




FiG. 4.-Same as Fig. 3, except that the mean transmission over the $50 \AA$ wavelength interval shortward of the redshifted $304 \AA$ line is that appropriate for $\tau_{\text {it Il }}=1.0$ (Tytler 1995). As before, model 1 has line blanketing as the dominant opacity, while in model 2 the diffuse IGM dominates. For model 1 (solid curves) the source spectral index $\alpha_{2}=1.5$, the background radiation produces $\eta=70$, the LF extends from $N_{1}=10^{13} \mathrm{~cm}^{-2}$ to $N_{u}=10^{16} \mathrm{~cm}^{-2}$, lines are velocity broadened with the PR distribution $f(b)$, and the scaled diffuse IGM density $\widetilde{\Omega}_{1 G M}=0.0084$. For model 2 (dashed curve) $\alpha_{a}=1.5$, the back ground radiation produces $\eta=70$, the $L F$ extends from $N_{1}=10^{13} \mathrm{~cm}^{-2}$ to $N_{k}=10^{16} \mathrm{~cm}^{-2}$, lines are thermally broadened with the PR distribution $f(b)$, and the scaled diffuse IGM density $\Omega_{\mathrm{IGM}}=0.0154$

transmission, $\langle T\rangle=0.37$, over a $50 \AA$ interval shortward of the edge. Here, also, the asymptotic optical depths are larger than the average optical depth due to the proximity effect. Thus, including the proximity effect markedly increases the amount of $\mathrm{He}$ II absorption which must be accounted for in $\mathrm{Q} 0302-003$. In the low-opacity case, it is actually difficult to construct a model in which the diffuse IGM opacity dominates the contribution from line blanketing. For models with $N_{l}=$ $10^{13} \mathrm{~cm}^{-2}$ only thermal broadening models may accomplish this.

\subsection{Limits on the IGM Density}

An adequate model for the observed He II absorption requires realistic parameters for the $\mathrm{LF}$ as well as the ionizing intensities of the individual quasar and background. One can calculate the contribution due to the GP optical depth of the diffuse IGM and thus provide an estimate of the IGM density $\left(\Omega_{\mathrm{GM}}\right)$. Our models include those dominated by both $\tau_{\mathrm{GP}}$ and $\tau_{\mathrm{LF}}$, but in most successful models there are comparable contributions from each source of opacity. With its relatively high line density, the PR model for the LF can easily reproduce $\tau_{\mathrm{He} \mathrm{II}}=1-2$ by line blanketing. The LF dominates the diffuse IGM near the $\mathrm{He}$ II $\lambda 304$ edge, but the reverse is true at shorter wavelengths. Although we have assumed that the diffuse IGM is photoionized by the same radiation as the Ly $\alpha$ clouds, it is possible that some of the diffuse medium is collisionally ionized. This caveat only strengthens our assertion that much of the observed $\mathrm{He}$ II decrement is the result of cloud line blanketing.

The choice of LF model strongly affects the results, both through filtering by the clouds of the source spectrum and line blanketing. There are even successful models in which the diffuse $\Omega_{I G M} \approx 0$. Consistent with Madau \& Meiksin (1994), we find that velocity broadening eliminates the need for the $\mathrm{H} \mathrm{I}$ clouds of extremely low column density $\left(N_{\mathrm{H} I} \approx 10^{-11} \mathrm{~cm}^{-2}\right)$ suggested by Jakobsen et al. (1994). Indeed, clouds of column density $N_{\mathrm{HI}} \lesssim 10^{12} \mathrm{~cm}^{-2}$ may not exist owing to constraints posed for large Ly $\alpha$ clouds by density contrast with the IGM.

For PR models in which we vary $N_{l}$ and $N_{u}$ and assume velocity broadening, Figures 5 and 6 show the scaled IGM density required to satisfy $\langle T\rangle \lesssim 0.18$ toward $\mathrm{Q} 0302-003$ with and without the proximity effect. For each curve, the hardness of the underlying spectrum $\left(\alpha_{s}\right)$ determines the contribution of line blanketing to the observed He II opacity and consequently the additional opacity supplied by the diffuse IGM. Since our assumed $\mathrm{He}$ II opacity, $\tau_{\mathrm{He} \text { II }} \geq 1.7$, is a lower limit (Jakobsen et al. 1994), the allowed range in $\boldsymbol{\Omega}_{\mathrm{IGM}}$ lies to the right of these curves. We also show the limits on $\Omega_{\mathrm{IGM}}$ expected for $\tau_{\mathrm{GP}}\left(\mathrm{H}_{\mathrm{I}}\right)=0.05-0.10$ at $z=3.29$ (Steidel \& Sargent 1987; Fang \& Crotts 1995). One can see from Figures 5 and 6 that the proximity effect increases the required value of $\Omega_{\text {IGM }}$. A conservative estimate of the LF contribution $\left(N_{1} \geq\right.$ $\left.10^{13} \mathrm{~cm}^{-2}\right)$ to $\mathrm{He}$ II line blanketing requires source spectra



FIG. 5,-Spectral index $x_{s}$ of ionizing sources and scaled diffuse IGM density $\left(\tilde{\Omega}_{I G M}\right)$ required to satisfy the observed $\tau_{H_{e} \| I} \geq 1.7$ in the spectrum of 00302-003 for different models of the LF distribution. Allowed values of $\widehat{\Omega}_{\mathrm{IGM}}$ lie on or above the solid or dashed lines and to the left of the two vertical dotted lines, which correspond to range of $\mathrm{H}, \mathrm{GP}$ limits $\tau_{\mathrm{GP}}\left(\mathrm{H}_{\mathrm{i}}\right)=0.05$ or 0.10 . Solid lines include the QSO proximity effect: dashed lines assume a metagalactic background only $\left(J_{-21}=1\right)$. We adopt the PR distributions in $N_{\mathrm{H}}$ and $b$-values with velocity-broadened lines, with $N_{i}\left(\mathrm{H}_{1}\right)=10^{13} \mathrm{~cm}^{-2}$ and $N_{u}\left(\mathrm{H}_{1}\right)$ equal to either $10^{15}$ or $10^{17} \mathrm{~cm}^{-2}$ as labeled. 


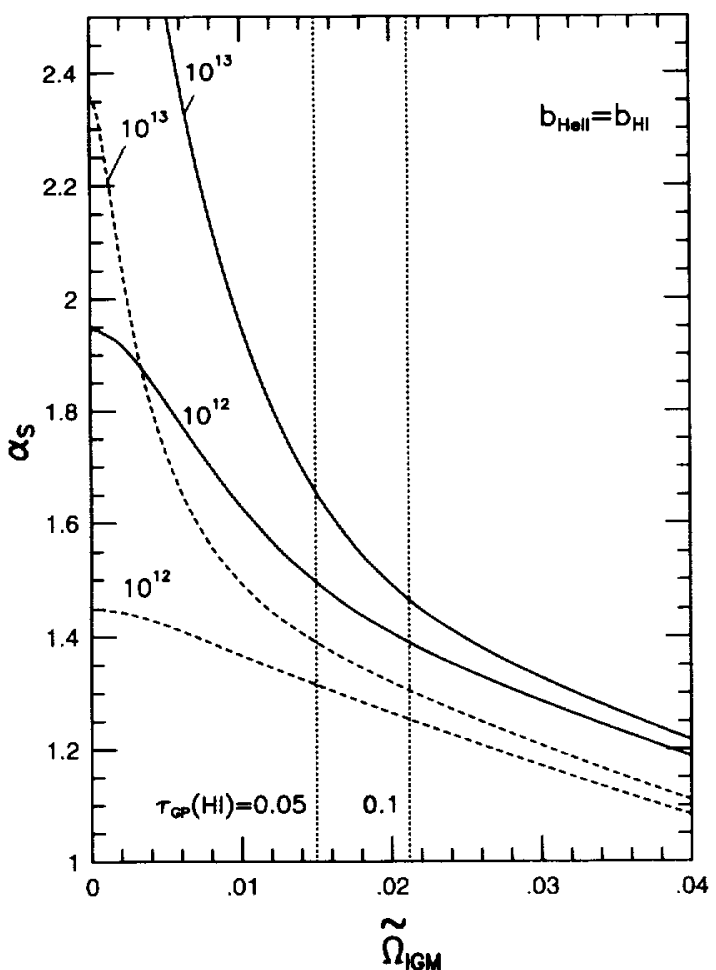

Fig. 6.--Same as Fig. 5 , for $\tau_{\mathrm{He}} \mathrm{l}=1.7$, except that the $\mathrm{H} \mathrm{I}$ column distribution has $N_{u}\left(\mathrm{H} \mathrm{I}_{1}\right)=10^{16} \mathrm{~cm}^{-2}$ and $N_{,}\left(\mathrm{H}_{\mathrm{H}}\right)$ is either $10^{12}$ or $10^{13} \mathrm{~cm}^{-2}$ as labeled.



Fig. 7,-Same as Fig. 6, except that $\tau_{\text {He II }}=1.0$ as observed by Tytler (1995).



Fig. 8.-Same as Fig. 6, except that $\tau_{\mathrm{He} \|}=\mathbf{3 . 0}$, and the $\mathbf{H} 1$ column distribution has $N(\mathrm{H} t)$ either $10^{12}$ or $10^{12.5} \mathrm{~cm}^{-2}$ as labeled.

with $\alpha_{s}>1.3-1.4$, even allowing the largest possible contribution from the IGM consistent with limits on $\tau_{\mathrm{GP}}(\mathrm{H} \mathrm{I})$. The inclusion of the quasar proximity effect significantly diminishes the allowed region of parameter space, increasing the minimum $\alpha_{s}$ possible by $\sim 0.2$ and restricting the possibility that line blanketing alone can account for the observed $\mathrm{He}$ absorption. However, it does not rule out pure line-blanketing models. Over much of the remaining parameter space, the proximity effect produces substantial transmission $(T>20 \%)$ from 1290 to $1302 \AA$, although the transmission is greatly diminished at $\lambda<1290 \AA$ if $\boldsymbol{\Omega}_{\mathrm{IGM}}$ is large. The proximity effect is most important for soft source spectra and models with thermally broadened lines.

Consider cases with LF models favorable for line blanketing (velocity-broadened lines and $N_{l}=10^{12} \mathrm{~cm}^{-2}$ ). Figure 6 shows that, even with the $\mathrm{He}$ II proximity effect included, one can construct models with no IGM provided that $\alpha_{s} \geq 1.9$. As noted earlier, this result derives primarily from the lessened sensitivity of line blanketing to the He Il proximity effect. A final point is that, except in the most extreme LF models, it is difficult to satisfy both the He II and $\mathrm{H}_{\mathrm{I}}$ GP limits with $\alpha_{\mathrm{s}}<$ 1.5. While it is too early to interpret these results as support for thermal bump models of AGN (Terasawa 1992), softening the EUV spectra of the background makes it easier to satisfy observational constraints. A substantial stellar component for the ionizing background (Madau \& Shull 1995) would also soften the EUV spectrum.

The recent report by Tytler et al. (1995) that $\tau_{\text {He II }} \approx 1$ at $z=3.185$ may conflict with the $\tau_{\mathrm{He} \text { II }} \gtrsim 1.7$ limit of Jakobsen et al. (1994). Possibly, Q1935-67 has more He II ionizing radiation, enhancing its proximity effect. If $\tau_{\mathrm{He} \mathrm{II}}=1$ turns out to be a typical value at $z>3$, models that include only line blanketing can easily account for the absorption. As Figure 7 shows, if 
$N_{l}=10^{13} \mathrm{~cm}^{-2}$ and $N_{u}=10^{16} \mathrm{~cm}^{-2}$, with velocity broadening one needs only $\alpha_{s}>1.8$ to produce the absorption without a diffuse IGM. If the proximity effect is not included, this requirement is relaxed to $\alpha_{s}>1.4$. If clouds with $N_{l}<10^{13}$ $\mathrm{cm}^{-2}$ are included, it becomes more difficult to avoid exceeding $\tau_{\mathrm{He} I I}=1$, assuming velocity broadening. A low value for the He II opacity may imply that the background sources are hard, $\alpha_{s}<1.2-1.4$.

We now argue that, if $\tau_{\mathrm{He} \mathrm{II}} \gtrsim 3$ at $z>3$, one requires both a substantial diffuse IGM and soft sources $\left(\alpha_{s}>2\right)$. The dashed (no $\mathrm{He}$ II proximity effect) lines in Figure 8 show that the sources must have very soft spectra if line blanketing alone is to account for the He II absorpion. If $N_{1}=10^{12} \mathrm{~cm}^{-2}$, then $\alpha_{s}$ must exceed 2; if $N_{l}=3 \times 10^{12} \mathrm{~cm}^{-2}$ then $\alpha_{s}>2.7$. If the proximity effect is included (solid lines in Fig. 8), there must be a substantial contribution from the diffuse IGM. Even if the sources were as soft as $\alpha_{s}=3$ (i.e., $\eta>10^{4}$ ), then $\Omega_{1 G M}$ would still exceed 0.005 . Further, if $\tau_{\mathrm{He}} \mathrm{Z} \gtrsim 3$, the H I GP constraints require $\alpha_{s}>2.2$.

\section{DISCUSSION}

Now that $\mathrm{He}$ II absorption observations are possible, what can be done to maximize their significance? First, observers should at tempt to resolve the He II edge at $(303.784 \AA)\left(1+z_{q}\right)$. Although difficult with the $H S T / F O C$ prism toward Q0302-003, this experiment could be performed toward this and selected other targets by current HST spectrographs (GHRS or FOS) and by the second-generation HST/STIS spectrograph. Second, better photon statistics and wavelength calibration can detect or further limit flux below the He II Lya edge and perhaps detect the proximity effect; such a detection would give a new estimate of $J_{-21}$. Improved measurements of the flux shortward of the $\mathrm{He}$ II edge toward Q0302-003 that resulted in values $\tau(\mathrm{He} \mathrm{II})=3-5$ would provide strong evidence for a diffuse IGM contribution. As Figure 8 shows, it is difficult for a pure line-blanketing model and sources with $\alpha_{s}<2.5$ to provide such a high opacity, especially if the He II proximity effect is included. Third, observations with better resolution could distinguish the relative contributions of the IGM and the LF to He II absorption by resolving the shape of the spectrum near the AGN redshift or by detecting gaps between the lines. Based on the modeled Ly $\alpha$ line density, we estimate that the $1250-1300 \AA$ region in $\mathrm{Q} 0302-003$ could contain 80-90 lines with $N\left(\mathrm{HI}_{\mathrm{l}}\right)>10^{13} \mathrm{~cm}^{-2}$, although the $\mathrm{He}$ II void inferred by Dobrzycki \& Bechtold (1991) between 1262 and $1275 \AA$ could reduce this estimate to $\sim 65$ lines. Even accounting for this void, 2-3 $\AA$ resolution should be sufficient to identify continuum gaps arising from Poisson fluctuations. Fourth, a direct measurement of the He line widths (from $\mathrm{He}$ I or He II) with the Far Ultraviolet Spectroscopic Explorer (FUSE) to compare their line widths to those of $\mathrm{H}$ I would eliminate a significant uncertainty in the line-blanketing model. Finally, a measurement of the systemic redshift of $\mathrm{Q} 0302-003$ from low-ionization lines is important to distinguish $z_{q}$ from $z_{\mathrm{em}}$ and better establish the magnitude of the quasar proximity effect. This measurement could perhaps be done in the $\mathrm{K}$ band from $\mathrm{H} \beta$ (at $2.084 \mu \mathrm{m}$ ) or [O $\mathrm{OII}]$ (at 2.125 and $2.146 \mu \mathrm{m}$ ).

Figures 5 through 8 indicate that it is quite possible that a substantial fraction of the baryon density inferred from big bang nucleosynthesis limits may reside in the diffuse IGM. Further, the latest estimates (Madau \& Shull 1995) of the baryon fraction contributed by the Ly $\alpha$ forest clouds, $\Omega_{\mathrm{LF}} \approx$ $0.01 h^{-1}$, may also make up a substantial fraction of the total baryon density. As a result, it is possible that the majority of the dark baryons are found in the diffuse IGM and Lyx forest clouds. As He II absorption measurements improve, the diffuse IGM density will be more accurately constrained. The possibility will then exist, at least for $z \sim 3$, that these estimates may be combined with those for the baryon densities in Lyman limit and damped Ly $\alpha$ clouds to account for all of the baryons.

The He II GP experiments will require significant amounts of observing time, either on the $H S T$ or on future ultraviolet missions such as ASTRO2 (HUT) or FUSE. However, the potential rewards are great. The principal gains of such measurements are the determination of the intensity and spectrum of the ionizing background, the nature of the Ly $\alpha$ line widths, and the definitive detection of the long-sought diffuse IGM.

This work was supported by the Astrophysical Theory Program at the University of Colorado (NASA grants NAGW-766 and NAGW-1479). We thank David Tytler and Piero Madau for useful discussions. J. M. S. thanks the Visitors Program at the Space Telescope Science Institute, where the final portion of this work was completed.

\section{REFERENCES}

Bajtlik, S., Duncan, R. C., \& Ostriker, J. P. 1988, ApJ, 327, 570

Bechtold, J. 1994, ApJS, 91,

Bechtold, J., Crotts, A., Fang, Y., \& Duncan, R. 1994, ApJ, 437, L83

Corbin, M. R. 1990, ApJ, 357, 346

Dobrzycki, A., \& Bechtold, J. 1991, ApJ, 377, L69

Espey, B. R., Carswell, R. F., Bailey, J. A., Smith, M. G., \& Ward, M. J. 1989, ApJ, 342, 666

Fang, Y., \& Crotts, A. 1995, ApJ, 440, 69

Francis, P. 1995, private communication

Gaskell, C. M. 1982, ApJ, 263,79

Giroux, M. L. 1992, Ph.D. thesis, Univ. of Texas

Giroux, M. L., \& Shapiro, P. R. 1995, ApJ, submitted

Gunn, J. E., \& Peterson, B. A. 1965, ApJ, 142, 1633 (GP)

Jakobsen, P., Boksenberg, A., Deharveng, J. M., Greenfield, P., Jedrzejewski, R., \& Paresce, F. 1994, Nature, 370,35

Madau, P 1995 private communication

Madau, P \& Meiksin A. 1994, ApJ, 433, L53

Madau, P \& Shull, J. M. 1995, ApJ, submitted

Miralda-Escudé, J. 1993, MNRAS, 262, 273

Miralda-Escudé, J., \& Ostriker, J. P. 1990, ApJ, 350, I

-1992 ApJ, 392, 15

Miralda-Escudé, J., \& Rees, M. J. 1994, MNRAS, 266, 343
Møller, P., \& Jakobsen, P. 1990, A\&A, 228, 299

Paresce, F., McKee, C. F. \& Bowyer, S. 1980, ApJ, 240, 387

Press, W. H., \& Rybicki, G. B. 1993, ApJ, 418, 585 (PR)

Rauch, M. Carswell, R. F Chaffee, F. H., Foltz C. B., Webb, J. K.. Weymann, R. J., Bechtold, J., \& Green, R. F. 1992, ApJ, 390, 387

Rauch, M., Carswell, R. F., Webb, J. K.. \& Weymann, R. J. 1993, MNRAS, 260, 589

Sargent, W. L. W. Steidel, C. C. \& Boksenberg, A. 1989, ApJS, 69, 703 (SSB)

Schneider. D. P Schmidt, M.\& Gunn, J. E. 1991, AJ, 101, 2004

Shapiro, P. R., Giroux, M. L., \& Babul, A. 1994, ApJ, 427, 25

Songaila, A., Hu, E. M., \& Cowie, L. L. 1995, preprint

Steidel, C. C., \& Sargent, W. L. W. 1987. ApJ, 318 , L11

Sutherland, R., \& Shull, J. M. 1995, ApJ, to be submitted

Terasawa, N. 1992, ApJ, 392, L15

Tytler, D., Fan, X.-M., Burles, S., Cottrell, L., David, C., Kirkman, D., \& Zuo. L. 1995 , in QSO Absorption Lines, Proc. ESO Workshop, ed. J. Bergeron, G. Meylan, \& J. Wampler (Heidelberg: Springer), in press

Tytier, D. \& Fan, X.-M. 1992, ApJS, 79, 1

Webb, J. K., Barcons, X., Carswell, R. F., \& Parnell, H. C. 1992. MNRAS, 255 319

Zheng, W., \& Davidsen, A. 1995, ApJ, 440. L53 
